\title{
Differential Inhibitory Activities of Four Plant Essential Oils on In Vitro Growth of Fusarium oxysporum f. sp. fragariae Causing Fusarium Wilt in Strawberry Plants
}

\author{
Jin Young Park ${ }^{1}$, Su Hyeon Kim ${ }^{1}$, Na Hee Kim ${ }^{1}$, Sang Woo Lee ${ }^{1}$, Yong-Chull Jeun ${ }^{2}$, and Jeum Kyu Hong ${ }^{1 *}$ \\ ${ }^{1}$ Department of Horticultural Science, Gyeongnam National University of Science and Technology (GNTech), Jinju \\ 52725, Korea \\ ${ }^{2}$ College of Applied Life Science, Faculty of Bioscience and Industry, The Research Institute for Subtropical Agriculture \\ and Biotechnology, Jeju National University, Jeju 63243, Korea
}

(Received on June 30, 2017; Revised on August 16, 2017; Accepted on August 21, 2017)

The objective of this study was to determine inhibitory activities of four volatile plant essential oils (cinnamon oil, fennel oil, origanum oil and thyme oil) on in vitro growth of Fusarium oxysporum f. sp. fragariae causing Fusarium wilt of strawberry plants. Results showed that these essential oils inhibited in vitro conidial germination and mycelial growth of $F$. oxysporum $f$. sp. fragariae in a dose-dependent manner. Cinnamon oil was found to be most effective one in suppressing conidial germination while fennel oil, origanum oil and thyme oil showed moderate inhibition of conidial germination at similar levels. Cinnamon oil, origanum oil and thyme oil showed moderate antifungal activities against mycelial growth at similar levels while fennel oil had relatively lower antifungal activity against mycelial growth. Antifungal effects of these four plant essential oils in different combinations on in vitro fungal growth were also evaluated. These essential oils demonstrated synergistic antifungal activities against conidial germination and mycelial growth of $F$. oxysporum $f$. sp. fragariae in vitro. Simultaneous application of origanum oil and thyme oil enhanced their antimicrobial activities against conidial germination and fungal mycelial growth. These results underpin that volatile plant es-

\footnotetext{
*Corresponding author.

Phone) +82-55-751-3251, FAX) +82-55-751-3257

E-mail) jkhong@gntech.ac.kr

(c) This is an Open Access article distributed under the terms of the Creative Commons Attribution Non-Commercial License (http:// creativecommons.org/licenses/by-nc/4.0) which permits unrestricted noncommercial use, distribution, and reproduction in any medium, provided the original work is properly cited.
}

Articles can be freely viewed online at www.ppjonline.org. sential oils could be used in eco-friendly integrated disease management of Fusarium wilt in strawberry fields.

Keywords : antifungal, Fusarium oxysporum f. sp. fragari$a e$, plant essential oil, synergistic, volatile

Handling Associate Editor : Jeon, Junhyun

Fusarium wilt by Fusarium oxysporum is one intractable soil-borne plant disease that causes devastating losses of many economically important horticultural crops, including banana, muskmelon, tomato and strawberry plants. Fusarium wilt has been continuously spreading in strawberry plant growing fields. Strawberry Fusarium wilt disease has been increasing reported in many countries in Europe, Asia and America recently (Arroyo et al., 2009; Dinler et al., 2016; Koike et al., 2009; Stanković et al., 2014). In South Korea, strawberry Fusarium wilt occurs in all plant growing seasons, including nursery and protected cropping field stages of several cultivars prevalently planted such as Akihime and Maehyang (Nam et al., 2005). Genetic diversity has been found in Korean isolates of $F$. oxysporum f. sp. fragariae depending on strawberry cultivation regions (Kim et al., 2017).

To control strawberry Fusarium wilt, a variety of approaches have been suggested, including cultural, biological and chemical methods. Planting disease-resistant cultivars against Fusarium wilt is the most promising approach for sustainable horticulture. Commercial strawberry cultivars resistant to Fusarium wilt have been analysed (Cho and Moon, 1984; Fang et al., 2012; Kim et al., 1982; Mori et al., 2005; Nam et al., 2005). Strawberry cultivar highly 
resistant to Fusarium wilt has been hardly reported worldwide. Cultivar Seolhyang is relatively resistant to Fusarium wilt compared to cultivar Maehyang (Lee et al., 2010). Cultivar Seolhyang is planted in more than $70 \%$ of strawberry fields in South Korea. This is dangerous because highly virulent isolates can overcome cultivar resistance. Although in vitro inhibitory effects of several chemical fungicides such as thiophanate-methyl on growth of Fusarium oxysporum f. sp. fragariae have been investigated, only limited number of these fungicides such as copper hydroxide have been used to control strawberry Fusarium wilt in fields (Cho and Moon, 1984; Kim et al., 1982). Supplement with organic matter and calcium cyanide during soil solarization can reduce Fusarium wilt before transplanting strawberry seedlings into fields (Nam et al., 2011). Eco-friendly biocontrol agents are needed to introduce strawberry plant cultures during growing seasons. In recent years, antagonistic bacteria (Bacillus velezensis, Streptomyces sp.) and symbiotic mycorrhizal fungus (Glomus mosseae) have been applied in strawberry growing fields to decrease Fusarium wilt (Cha et al., 2016; Matsubara et al., 2004; Nam et al., 2009). Recent progresses in management strategies of strawberry Fusarium wilt have been reviewed by Koike and Gordon (2015).

Plant essential oils with a broad spectrum of antimicrobial activity have been applied to manage fungal and bacterial diseases of many vegetables. They might be used as green pesticides in integrated pest management programmes (Bajpai et al., 2011; Koul et al., 2008; Sivakumar and Bautista-Baños, 2014). Our previous studies have shown that different plant essential oils can efficiently control pepper fruit anthracnose caused by Colletotrichum gloeosporioides fungus and tomato plant wilting caused by Ralstonia pseudosolanacearum bacterium (Hong et al., 2015; Lee et al., 2012). Applying plant essential oils has been strongly suggested as an alternative method to control Fusarium wilt of banana, muskmelon and tomato plants (Bowers and Locke, 2000; Monteiro et al., 2013; Sharma et al., 2017). However, using plant essential oils to control Fusarium wilt of strawberry has not been reported yet.

Therefore, the objective of this study was to evaluate inhibiting activities of four plant essential oils (cinnamon oil, fennel oil, origanum oil and thyme oil) on in vitro growth of $F$. oxysporum f. sp. fragariae. These four plant essential oils have been reported to possess antifungal activities against conidial germination and/or mycelial germination of a variety of phytopathogenic fungi at relatively low concentrations (Daferera et al., 2003; Monteiro et al., 2013; Soylu et al., 2007, 2010). In addition, synergistic effect of these plant essential oils in combination on in vitro fungal growth was also evaluated in this study.

\section{Materials and Methods}

Fungal cultures. F. oxysporum f. sp. fragariae isolate SFW-10 used in the present study was isolated from naturally infected strawberry plants (cv. Akihime) grown in Hadong-gun, Gyeongnam, South Korea in 2010. This fungus was cultured on $1 / 2$ potato dextrose agar (PDA) media at $25^{\circ} \mathrm{C}$. To obtain conidial suspension, mycelia were streaked onto culture media and incubated for 14 days. Quarterly diluted sterile potato dextrose broth (PDB) was poured onto the 14 day-old fungal culture. The concentration of conidial suspension was adjusted to $5 \times 10^{5}$ conidia/ $\mathrm{ml}$. To prepare mycelial plugs, mycelial discs $(5 \mathrm{~mm}$ in diameter) were inoculated at the center of the media. Fresh mycelial discs (5 $\mathrm{mm}$ in diameter) were cut from the growing edge of 7 day-old PDA cultures.

Preparation of paper disc harbouring plant essential oils. Cinnamon oil and thyme oil were purchased from Sigma-Aldrich Co., Ltd (St. Louis, MO, USA). Fennel oil and origanum oil were purchased from Tokyo Chemical Industry Co., Ltd (Tokyo, Japan). Each viscous plant essential oil concentrate was diluted to 5 or $20 \%(\mathrm{v} / \mathrm{v})$ solution in $95 \%$ ethanol for easy handling. Increasing doses of each plant essential oil $(0,0.1,0.2,0.5,1,2$ and $5 \mu 1 /$ disc $)$ were dropped onto sterile paper disc ( $8 \mathrm{~mm}$ in diameter) (Toyo Roshi Kaisha, Ltd, Tokyo, Japan) and 95\% ethanol was additionally laid to make the same loading volume (50 $\mu 1)$ in each paper disc. These paper discs were air-dried for $20 \mathrm{~min}$ at room temperature to evaporate ethanol on a clean bench.

Inhibitory effect of volatile plant essential oils on in vitro conidial germination and mycelial growth. Volatile antifungal activities of these four plant essential oils against in vitro conidial germination and mycelial growth were evaluated using method described in our previous study (Hong et al., 2015) with slight modifications. Briefly, for conidial germination-inhibiting assays, four conidial suspension drops (20 $\mu 1$ each) prepared in $1 / 4 \mathrm{PDB}$ on glass slides were treated with plant essential oils for 10 $\mathrm{h}$ at $25^{\circ} \mathrm{C}$ in plastic square dishes $(100 \mathrm{~mm} \times 100 \mathrm{~mm} \times$ $13 \mathrm{~mm}$ ). Conidia were stained with $1 \mu 1$ of lactophenoltrypan blue solution and conidial suspensions were covered by coverslips before observation under a microscope. For mycelial growth-inhibiting assays, fungal mycelial plugs (5 $\mathrm{mm}$ in diameter) were inoculated onto the centre of $10 \mathrm{ml}$ of PDA media in Petri dishes $(85 \mathrm{~mm}$ in diameter, $13 \mathrm{~mm}$ 
in height). Fungal colony diameters were measured at 6 days after inoculation onto PDA media at $25^{\circ} \mathrm{C}$. Diameters of fungal colonies treated with plant essential oils were compared to those of untreated colonies and expressed as percentage $(\%)$.

Simultaneous treatment with different plant essential oils. Different plant essential oils were applied simultaneously to determine their synergistic efficacies against in vitro conidial germination and mycelial growth of $F$. oxysporum f. sp. fragariae. Paper discs containing 0.5 and $1 \mu \mathrm{l} /$ disc of each plant essential oil were attached to the inside plate cover singly or concurrently with other paper disc(s) for conidial germination and mycelial growth inhibition assays, respectively. Conidial germination and mycelial growth were evaluated at $10 \mathrm{~h}$ and 6 days after incubation at $25^{\circ} \mathrm{C}$ with different combinations of these plant essential oils described above.

Statistical analyses. Data were subjected to analysis of variance using SAS version 9.1 (SAS Institute, Inc., Cary, NC, USA). Means were separated by least significant difference (LSD) test at $P<0.05$. Graphing was prepared using SigmaPlot 10.0 (Systat Software, Inc., San Jose, CA, USA).

\section{Results}

Different antifungal efficacies of four plant essential oils against in vitro conidial germination of $F$. oxysporum f. sp. fragariae. The four plant essential oils tested in this study showed different antifungal efficacies against in vitro conidial germination of $F$. oxysporum $\mathrm{f}$. sp. fragariae in dose-dependent manners (Fig. 1). Treatment with cinnamon oil drastically suppressed conidial germination at the lowest dose used $(0.1 \mu \mathrm{l} / \mathrm{disc})$. Conidial germination was completely inhibited by cinnamon oil at $0.2 \mu \mathrm{l} / \mathrm{disc}$. Fennel oil was less effective in reducing conidial germination compared to cinnamon oil. It failed to completely suppress conidial germination even at the highest dose used in this study ( $5 \mu \mathrm{l} / \mathrm{disc})$. Origanum oil and thyme oil demonstrated moderate antifungal activities against conidial germination at similar levels. Both of them inhibited conidial germination in a dose-dependent manner. Increasing concentrations of origanum oil and thyme oil concentrations resulted in gradual decreases of conidial germination. No germination was found in conidia treated with $5 \mu \mathrm{l} /$ disc of thyme oil.

Different antifungal efficacies of four plant essential oils against in vitro mycelial growth of $F$. oxysporum $f$.

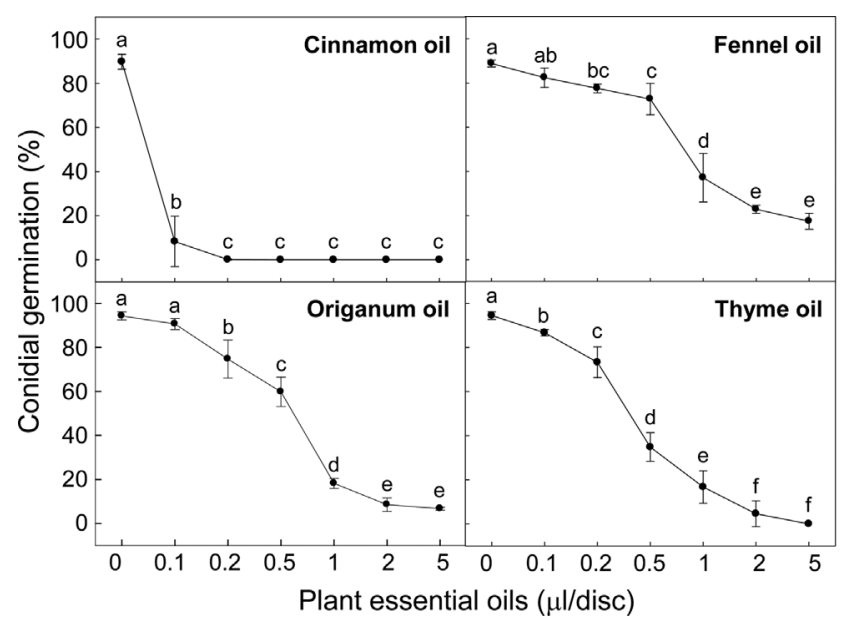

Fig. 1. Inhibition of conidial germination of Fusarium oxysporum f. sp. fragariae by four different volatile plant essential oils (cinnamon oil, fennel oil, origanum oil and thyme oil). Germinated conidia of $F$. oxysporum $\mathrm{f}$. sp. fragariae SFW-10 on glass slides after treatment with different doses $(0,0.1,0.2,0.5,1,2$ and $5 \mu \mathrm{l} /$ disc) of each volatile essential oil were observed under a light microscope. Relative conidial germination (\%) inhibited by different doses of each essential oil was shown as percentage of conidial germination compared to control. Error bars represent standard errors of means of four independent experimental replications. Means followed by the same letter are not significantly different at $5 \%$ level by least significant difference test. The same letter above bars represented no significant difference between treatments.

sp. fragariae. All four plant essential oils also possessed antifungal activities against mycelial growth of $F$. oxysporum f. sp. fragariae in a dose-dependent manner (Fig. 2). Cinnamon oil, origanum oil and thyme oil showed similarly elevated mycelial growth-inhibiting activities with increasing doses. Antifungal efficacy of cinnamon oil at 0.5 $\mu \mathrm{l} /$ disc was approximately $89.4 \%$ compared to untreated control. Increasing its dose to $1 \mu \mathrm{l} /$ disc did not augment its antifungal activity. However, relatively higher doses of cinnamon oil at $2 \mu \mathrm{l} / \mathrm{disc}$ and $5 \mu \mathrm{l} /$ disc resulted in significant reduction in mycelial growth (to approximately $60.0 \%$ and $6.9 \%$, respectively). At least $0.2 \mu \mathrm{l} /$ disc of origanum oil and thyme oil was needed to inhibit mycelial growth. Higher concentrations reduced mycelial growth more effectively. At the highest dose $(5 \mu \mathrm{l} /$ disc $)$, origanum oil and thyme oil completely inhibited mycelial growth. By contrast, fennel oil had relatively lower antifungal activity with limited ability to arrest mycelial growth with increasing doses. Even at the highest dose $(5 \mu \mathrm{l} /$ disc $)$, it only slightly reduced mycelial growth (approximately $84.4 \%$ compared to the untreated control). 


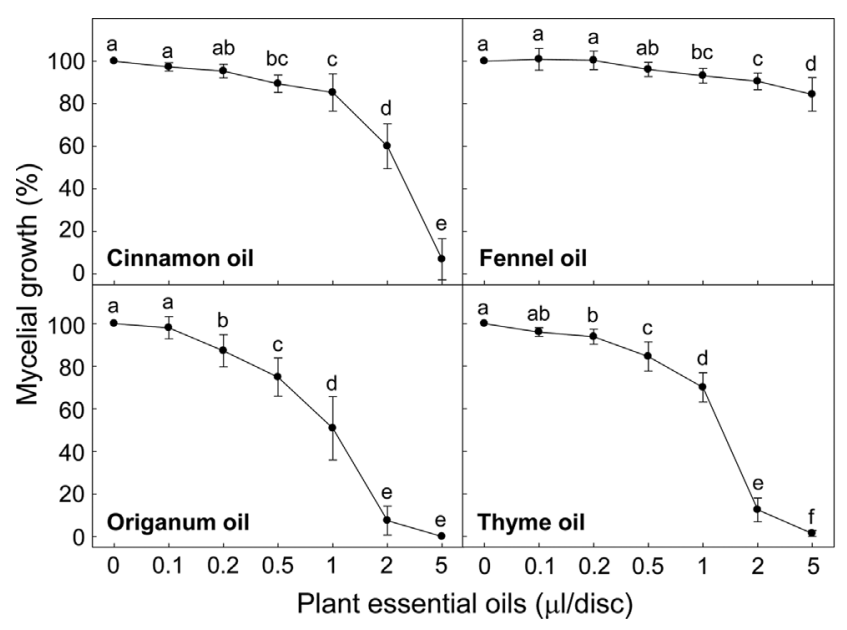

Fig. 2. Mycelial growth of Fusarium oxysporum f. sp. fragariae inhibited by four volatile plant essential oils (cinnamon oil, fennel oil, origanum oil and thyme oil). Colony formation of $F$. oxysporum f. sp. fragariae SFW-10 on PDA media after treatment with different doses $(0,0.1,0.2,0.5,1,2$ and $5 \mu 1 /$ disc $)$ of each volatile essential oil was determined. Relative mycelial growth inhibited by different doses of each essential oil is shown as percentage of fungal colonies on PDA. Error bars represent standard errors of means of four independent experimental replications. Means followed by the same letter are not significantly different at $5 \%$ level by least significant difference test. The same letter above bars represented no significant difference between treatments.

Synergistic antifungal effects of plant essential oils in combination on $F$. oxysporum f. sp. fragariae growth. Different combinations of these four plant essential oils were applied to investigate whether they might have synergism in suppressing conidial germination and mycelial growth of F. oxysporum f. sp. fragariae (Fig. 3, 4). Several combinations of these plant essential oils synergistically suppressed conidial germination (Fig. 3A). Treatment with cinnamon oil alone almost completely suppressed conidial germination. Additional treatment with other plant essential oils did not significantly increase the high suppressive activity of cinnamon oil. Three different combinations of two different essential oils (fennel oil + origanum oil, fennel oil + thyme oil, origanum oil + thyme oil) significantly increased their antifungal activities against conidial germination compared to any single plant essential oil treatment. No additional increase in antifungal activity against conidial germination was found by mixing three or four plant essential oils (Fig. 3B).

Enhanced suppressive activities against fungal mycelial growth were demonstrated by combined treatments with different plant essential oils (Fig. 4). After treatments with different combinations of essential oils, colony formation
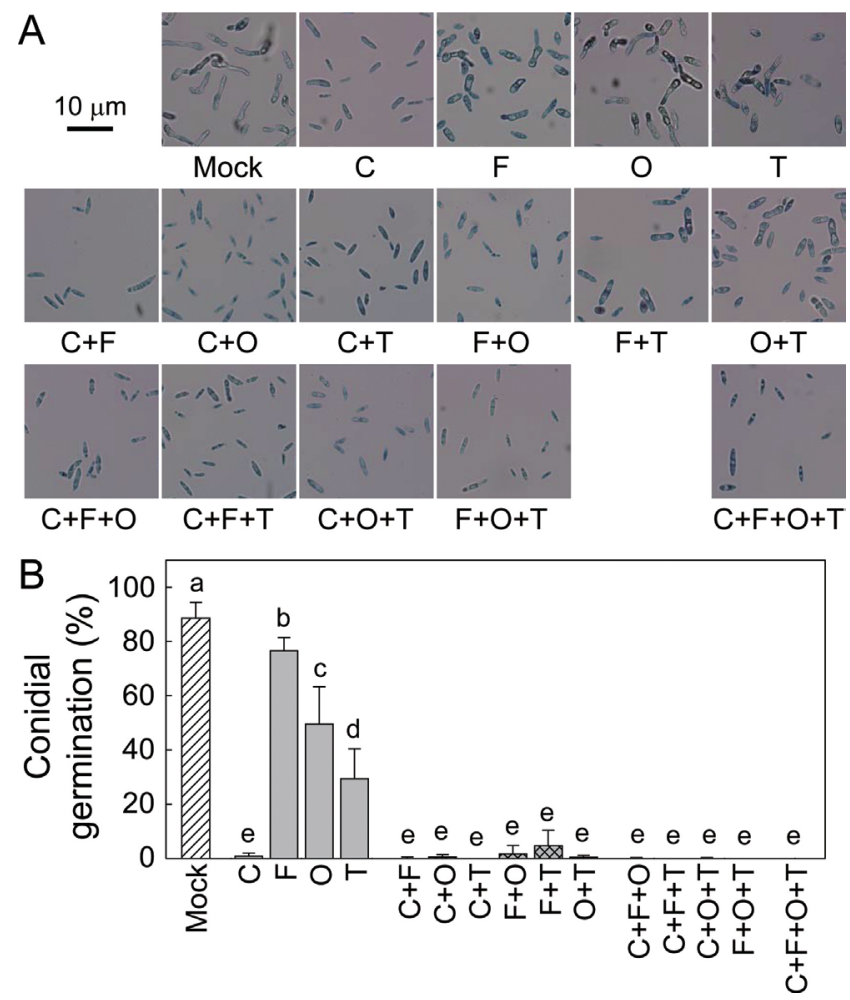

Fig. 3. Combination effect of four plant essential oils (cinnamon oil (C), fennel oil (F), origanum oil $(\mathrm{O})$ and thyme oil $(\mathrm{T})$ ) on their inhibitory activities against conidial germination of Fusarium oxysporum f. sp. fragariae. (A) Conidial germination at $10 \mathrm{~h}$ after incubation with or without plant essential oil treatment. Germinated conidia of $F$. oxysporum f. sp. fragariae SFW-10 on glass slides after treatment with $0.5 \mu \mathrm{l} /$ disc of each volatile essential oil were observed under a light microscope. (B) Relative conidial germination (\%) inhibited by different combinations of plant essential oils was shown as percentage of conidial germination compared to control. Error bars represent standard errors of means of four independent experimental replications. Means followed by the same letter are not significantly different at $5 \%$ level by least significant difference test. The same letter above bars represented no significant difference between treatments.

on PDA was demonstrated at 6 days after culture (Fig. 4A). Cinnamon oil treatment alone slightly reduced mycelial growth. Additional application with fennel oil failed to enhance its antifungal activity. However, addition application with origanum oil or thyme oil accelerated mycelial growth restriction caused by cinnamon oil. Single treatment with origanum oil or thyme oil drastically suppressed mycelial growth. Combined treatment with fennel oil and origanum oil showed synergistic effect on the suppression of mycelial growth compared to single treatment. Triple treatment with cinnamon oil, fennel oil and origanum oil resulted 

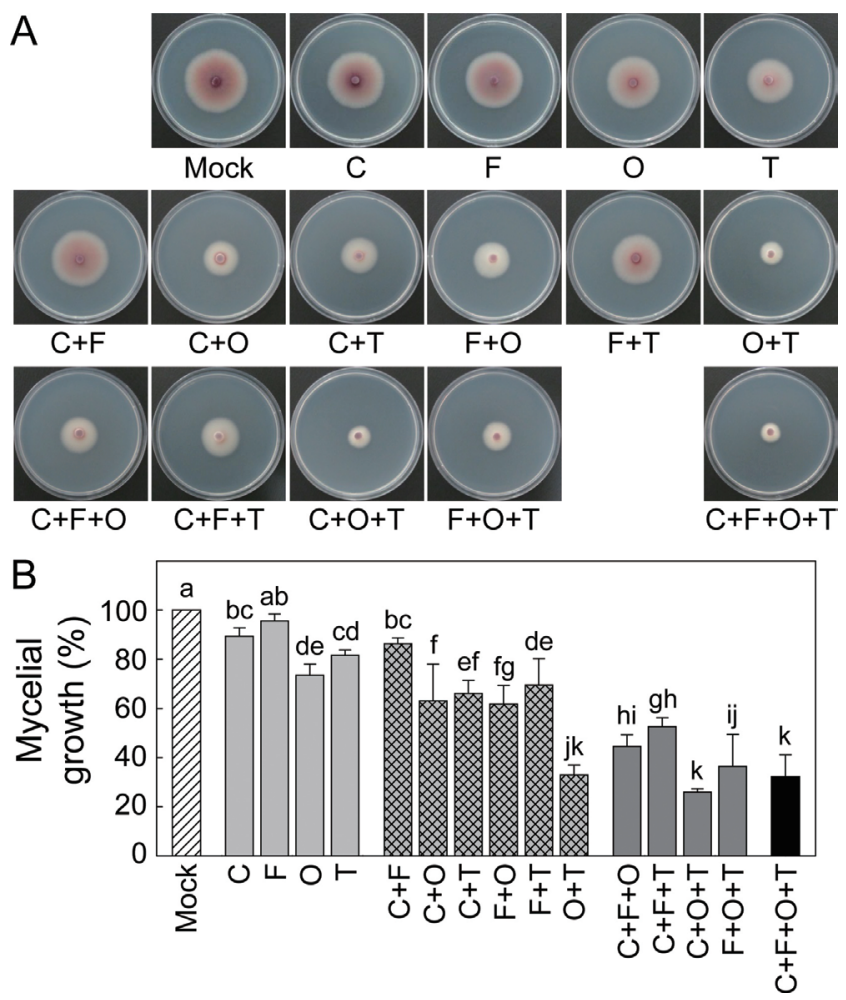

Fig. 4. Combination effect of four plant essential oils (cinnamon oil (C), fennel oil (F), origanum oil (O) and thyme oil (T)) on their inhibitory activities against mycelial growth of Fusarium oxysporum f. sp. fragariae. (A) Colony formation of F. oxysporum f. sp. fragariae SFW-10 on PDA media after treatment with $1 \mu \mathrm{l} /$ disc of each volatile essential oil. Photos were taken at 6 days after culture at $25^{\circ} \mathrm{C}$. (B) Relative mycelial growth inhibited by different combinations of plant essential oils shown as percentage of fungal colonies on PDA. Error bars represent standard errors of means of four independent experimental replications. Means followed by the same letter are not significantly different at $5 \%$ level by least significant difference test. The same letter above bars represented no significant difference between treatments.

in more reduction of mycelial growth. Antifungal activity against mycelial growths by triple treatment with cinnamon oil, origanum oil and thyme oil was similar to that by dual treatment with origanum oil and thyme oil. Triple treatment with cinnamon oil, fennel oil and thyme oil exerted additive antifungal activity compared to treatment with a combination of any two plant essential oils. Fennel oil treatment did not enhance the antifungal activity of dual treatment with origanum oil and thyme oil. Antifungal activity with a combination of all four essential oils was not significantly different from that with a combination of origanum and thyme oil (Fig. 4B).

\section{Discussion}

Due to their antifungal activities, plant essential oils have been applied in eco-friendly postharvest management to extend transportation and storage periods as well as shelf life of strawberry fruits in markets (Bhaskara Reddy et al., 1998; Nabigol and Morshedi, 2011). Several fungal species such as Botrytis cinerea, Rhizopus stolonifer and Aspergillus niger frequently deteriorating strawberry fruit quality have been controlled by various plant essential oils. However, control method to mitigate Fusarium wilt occurrence in strawberry plant culture fields is limited in spite of growing concerns of its worldwide spread. Antifungal activities of several plant essential oils against $F$. oxysporum causing Fusarium wilt in a variety of crop species have been evaluated. Clove oil has been found to be more effective in inhibiting in vitro conidial germination and mycelial growth of $F$. oxysporum f. sp. lycopersici causing tomato Fusarium wilt, compared to essential oils from lemongrass, mint, or eucalyptus plants (Sharma et al., 2017). Tomato Fusarium wilt disease has been efficiently reduced by treating soil mixtures with aqueous emulsion of clove oil (La Torre et al., 2016; Sharma et al., 2017). In this study, different antifungal activities of four plant essential oils against strawberry fungus $F$. oxysporum $\mathrm{f}$. sp. fragariae were demonstrated in vitro. Our results suggest that these essential oils might be useful to reduce Fusarium wilt in strawberry growing fields.

These four plant essential oils (cinnamon oil, fennel oil, origanum oil and thyme oil) tested in this study showed different antifungal efficacies against in vitro conidial germination and mycelial growth of $F$. oxysporum $\mathrm{f}$. sp. fragariae. Cinnamon oil was found to be the most effective one in suppressing conidial germination of $F$. oxysporum $\mathrm{f}$. sp. fragariae among these four plant essential oils. Origanum oil and thyme oil were more effective in reducing mycelial growth compared to the other two essential oils. These results suggest that various plant essential oils have different antifungal activities against conidial germination and mycelial growth. Cinnamon oil has been widely used to inhibit the growth of various phytopathogenic fungi. It has been reported that conidial germination and mycelial growth of $F$. oxysporum f. sp. cubense can be significantly suppressed by cinnamon oil, resulting in decreased panama disease in banana seedlings (Monteiro et al., 2013). Therefore, cinnamon oil treatment might be useful to halt early development of Fusarium wilt in strawberry fields via inhibiting conidial germination in soil environments. 
Origanum oil and thyme oil have not been applied to control $F$. oxysporum fungus yet. Both origanum oil and thyme oil can reduce spore germination and mycelial growth of postharvest destructive fungus Monilinia fructicola causing brown rot in stone fruits, including peaches and plums (Lazar-Baker et al., 2011). It has been reported that volatile origanum oil treatment can inhibit conidial germination, germ tube elongation and mycelial growth of grey mould fungus Botrytis cinerea (Soylu et al., 2010). Grey mould occurrence in tomato plants grown under greenhouse conditions can also be reduced via curative and protective effects of origanum oil (Soylu et al., 2010). Thyme oil can reduce in vitro growth of Phytophthora infestans and late blight of tomato plants in growing fields (El-Mohamedy and Abd-El-latif, 2015). Dose-dependent mycelial inhibition of $F$. oxysporum $\mathrm{f}$. sp. fragariae by origanum oil and thyme oil found in this study suggest that they might be useful to manage Fusarium wilt of strawberry plants.

Commercial products containing different plant essential oils as a component have been available for crop protection. It has been reported that combined treatment with rosemary oil, clove oil and thyme oil can reduce tomato Fusarium wilt much more efficiently in comparison with control effect with single treatment by clove oil, thyme oil, or rosemary oil (La Torre et al., 2016). This provides evidence that different plant essential oils can be used simultaneously to enhance their antifungal activity and crop protection efficacies. In the present study, combined treatment with origanum oil and thyme oil $(1: 1, \mathrm{vol} / \mathrm{vol})$ resulted in the best synergistic effect against mycelial growth of $F$. oxysporum f. sp. fragariae than other combinations. However, antifungal activities of origanum oil and thyme oil mixture at different ratios need to be elucidated to increase their antifungal efficacy.

To apply plant essential oils into crop fields practically, side effects including phytotoxicity should be considered. It has been shown that excess cinnamon oil can result in severe tissue damage, leading to detached tomato leaves (Lee et al., 2012). Dose-dependent phytotoxicities of soildrenching clove oil to cucumber, muskmelon, pepper and tomato seedlings before transplanting have also been reported (Meyer et al., 2008). Therefore, phytotoxic effect of plant essential oils should be defined before applying them in strawberry fields regarding their dosage, frequency and application method as well as plant cultivar, age and organs.

In summary, our results revealed that the four plant essential oils tested in this study could efficiently suppress conidial germination and mycelial growth of $F$. oxysporum f. sp. fragariae in vitro. These effects were more evident when two plant essential oils were mixed together compared to single plant essential oil treatment. Therefore, developing commercial products containing several plant essential oils might be promising as eco-friendly strategy to control strawberry Fusarium wilt.

\section{Acknowledgments}

This research was supported by Gyeongnam National University of Science and Technology (GNTech) Grant 2016 to J. K. H.

\section{References}

Arroyo, F. T., Llergo, Y., Aguado, A. and Romero, F. 2009. First report of Fusarium wilt caused by Fusarium oxysporum on strawberry in Spain. Plant Dis. 93:323.

Bajpai, V. K., Kang, S., Xu, H., Lee, S.-G., Baek, K.-H. and Kang, S. C. 2011. Potential roles of essential oils on controlling plant pathogenic bacteria Xanthomonas species: a review. Plant Pathol. J. 27:207-224.

Bhaskara Reddy, M. V., Angers, P., Gosselin, A. and Arul, J. 1998. Characterization and use of essential oil from Thymus vulgaris against Botrytis cinerea and Rhizopus stolonifer in strawberry fruits. Phytochemistry 47:1515-1520.

Bowers, J. H. and Locke, J. C. 2000. Effect of botanical extracts on the population density of Fusarium oxysporum in soil and control of Fusarium wilt in the greenhouse. Plant Dis. 84:300-305.

Cha, J.-Y., Han, S., Hong, H.-J., Cho, H., Kim, D., Kwon, Y., Kwon, S.-K., Crüsemann, M., Lee, Y. B., Kim, J. F., Giaever, G., Nislow, C., Moore, B. S., Thomashow, L. S., Weller, D. M. and Kwak, Y.-S. 2016. Microbial and biochemical basis of a Fusarium wilt-suppressive soil. ISME J. 10:119-129.

Cho, C. T. and Moon, B. J. 1984. Studies on the wilt of strawberry caused by Fusarium oxysporum f. sp. fragariae in Korea. Kor. J. Plant Protect. 23:74-81.

Daferera, D. J., Ziogas, B. N. and Polissiou, M. G. 2003. The effectiveness of plant essential oils on the growth of Botrytis cinerea, Fusarium sp. and Clavibacter michiganensis subsp. michiganensis. Crop Protect. 22:39-44.

Dinler, H., Benlioglu, S. and Benlioglu, K. 2016. Occurrence of Fusarium wilt caused by Fusarium oxysporum on strawberry transplants in Aydin province in Turkey. Australas. Plant Dis. Notes 11:10.

El-Mohamedy, R. S. R. and Abd-El-latif, F. M. 2015. Field application of humic acid and thyme essential oil for controlling late blight disease of tomato plants under field conditions. Asian J. Plant Pathol. 9:167-174.

Fang, X., Phillips, D., Verheyen, G., Li, H., Sivasithamparam, K. and Barbetti, M. J. 2012. Yields and resistance of strawberry cultivars to crown and root diseases in the field, and cultivar responses to pathogens under controlled environment condi- 
tions. Phytopathol. Mediterr. 51:69-84.

Hong, J. K., Yang, H. J., Jung, H., Yoon, D. J., Sang, M. K. and Jeun, Y.-C. 2015. Application of volatile antifungal plant essential oils for controlling pepper fruit anthracnose by Colletotrichum gloeosporioides. Plant Pathol. J. 31:269-277.

Kim, C. H., Seo, H. D., Cho, W. D. and Kim, S. B. 1982. Studies on varietal resistance and chemical control to the wilt of strawberry caused by Fusarium oxysporum. Kor. J. Plant Protect. 21:61-67 (in Korean).

Kim, J.-S., Kang, N. J., Kwak, Y.-S. and Lee, C. 2017. Investigation of genetic diversity of Fusarium oxysporum $\mathrm{f}$. sp. fragariae using PCR-RFLP. Plant Pathol. J. 33:140-147.

Koike, S. T. and Gordon, T. R. 2015. Management of Fusarium wilt of strawberry. Crop Protect. 73:67-72.

Koike, S. T., Korkpatrick, S. C. and Gordon, T. R. 2009. Fusarium wilt of strawberry caused by Fusarium oxysporum in California. Plant Dis. 93:1077.

Koul, O., Walia, S. and Dhaliwal, G. S. 2008. Essential oils as green pesticides: potential and constraints. Biopestic. Int. 4:63-84.

La Torre, A., Caradonia, F., Matere, A. and Battaglia, V. 2016. Using plant essential oils to control Fusarium wilt in tomato plants. Eur. J. Plant Pathol. 144:487-496.

Lazar-Baker, E. E., Hetherington, S. D., Ku, V. V. and Newman, S. M. 2011. Evaluation of commercial essential oil samples on the growth of postharvest pathogen Monilinia fructicola (G. Winter) Honey. Lett. Appl. Microbiol. 52:227-232.

Lee, J. N., Lim, J. S., Lee, J. G., Nam, C. W., Kim, K. D., Lee, E. H. and Yeoung, Y. R. 2010. Influence of the differences in altitude during raising seedlings on daughter plant characteristics and subsequent strawberry production. Kor. J. Hort. Sci. Technol. 28:540-544 (in Korean).

Lee, Y. H., Choi, C. W., Kim, S. H., Yun, J. G., Chang, S. W., Kim, Y. S. and Hong, J. K. 2012. Chemical pesticides and plant essential oils for disease control of tomato bacterial wilt. Plant Pathol. J. 28:32-39.

Matsubara, Y., Hirano, I., Sassa, D. and Koshikawa, K. 2004. Increased tolerance to Fusarium wilt in mycorrhizal strawberry plants raised by capillary watering methods. Seibutsu Kankyo Chosetsu 42:185-191.

Meyer, S. L. F., Lakshman, D. K., Zasada, I. A., Vinyard, B. T. and Chitwood, D. J. 2008. Phytotoxicity of clove oil to vegetable crop seedlings and nematotoxicity to root-knot nematodes. HortTechnology 18:631-638.
Monteiro, F. P., Ferreira, L. C., Silva, J. L., Pacheco, L. P. and Souza, P. E. 2013. Influence of plant extracts and essential oils against panama disease (Fusarium oxysporum f. sp. cubense) in banana seedlings. J. Agric. Sci. 5:63-74.

Mori, T., Kitamura, H. and Kuroda, K. 2005. Varietal differences in Fusarium wilt - resistance in strawberry cultivars and the segregation of this trait in $\mathrm{F}_{1}$ hybrids. J. Jpn. Soc. Hortic. Sci. 74:57-59.

Nabigol, A. and Morshedi, H. 2011. Evaluation of the antifungal activity of the Iranian thyme essential oils on the postharvest pathogens of strawberry fruits. Afr. J. Biotechnol. 10:98649869.

Nam, M. H., Jung, S. K., Kim, N. G., Yoo, S. J. and Kim, H. G. 2005. Resistance analysis of cultivars and occurrence survey of Fusarium wilt on strawberry. Res. Plant Dis. 11:35-38 (in Korean).

Nam, M. H., Kim, H. S. and Kim, H. G. 2011. Control of Fusarium wilt of the strawberry caused by Fusarium oxysporum f. sp. fragariae of solarization with compost and calcium cyanamide application. Res. Plant Dis. 17:32-37 (in Korean).

Nam, M. H., Park, M. S., Kim, H. G. and Yoo, S. J. 2009. Biological control of strawberry Fusarium wilt caused by Fusarium oxysporum f. sp. fragariae using Bacillus velezensis BS87 and RK1 formulation. J. Microbiol. Biotechnol. 19:520-524.

Sharma, A., Rajendran, S., Srivastava, A., Sharma, S. and Kundu, B. 2017. Antifungal activities of selected essential oils against Fusarium oxysporum f. sp. lycopersici 1322, with emphasis on Syzygium aromaticum essential oil. J. Biosci. Bioeng. 123:308-313.

Sivakumar, D. and Bautista-Baños, S. 2014. A review on the use of essential oils for postharvest decay control and maintenance of fruit quality during storage. Crop Protect. 64:27-37.

Soylu, E. M., Kurt, Ș. and Soylu, S. 2010. In vitro and in vivo antifungal activities of the essential oils of various plants against tomato grey mould disease agent Botrytis cinerea. Int. J. Food Microbiol. 143:183-189.

Soylu, S., Yigitbas, H., Soylu, E. M. and Kurt, Ş. 2007. Antifungal effects of essential oils from oregano and fennel on Sclerotinia sclerotiorum. J. Appl. Microbiol. 103:1021-1030.

Stanković, I., Ristić, D., Vučurović, A., Milojević, K., Nikolić, D., Krstić, B. and Bulajić, A. 2014. First report of Fusarium wilt of strawberry caused by Fusarium oxysporum in Serbia. Plant Dis. 98:1435. 